\title{
LASIK X PRK após cirurgia de descolamento de retina
}

\author{
LASIK X PRK after retinaldetachment surgery
}

\author{
Adriano Jorge Mattoso Rodovalho ${ }^{1}$ \\ Marcelo Guimarães Brandão Rego ${ }^{1}$ \\ João J. Nassaralla Júnior ${ }^{2}$ \\ Belquiz Rodrigues do Amaral Nassaralla ${ }^{3}$
}

Estudo realizado no Instituto de Olhos de Goiânia.

${ }^{1}$ Médico residente do Instituto de Olhos de Goiânia.

${ }^{2}$ Médico Oftalmologista do Departamento de Retina e

Vítreo do Instituto de Olhos de Goiânia (IOG); Doutorando em Oftalmologia pela Universidade Federal de Minas Gerais; Doutorando em Medicina pela Universidade de Brasília.

${ }^{3}$ Médica Oftalmologista do Departamento de Córnea e Cirurgia Refrativa do Instituto de Olhos de Goiânia (IOG); Doutora em Oftalmologia pela Universidade Federal de Minas Gerais.

Endereço para correspondência: Rua Antonio Costa Carvalho, 255/71 - Campinas (SP) CEP 13024-050

E-mail: adriano_jorge@uol.com.br

Recebido para publicação em 01.02.2002 Aceito para publicação em 14.08.2002

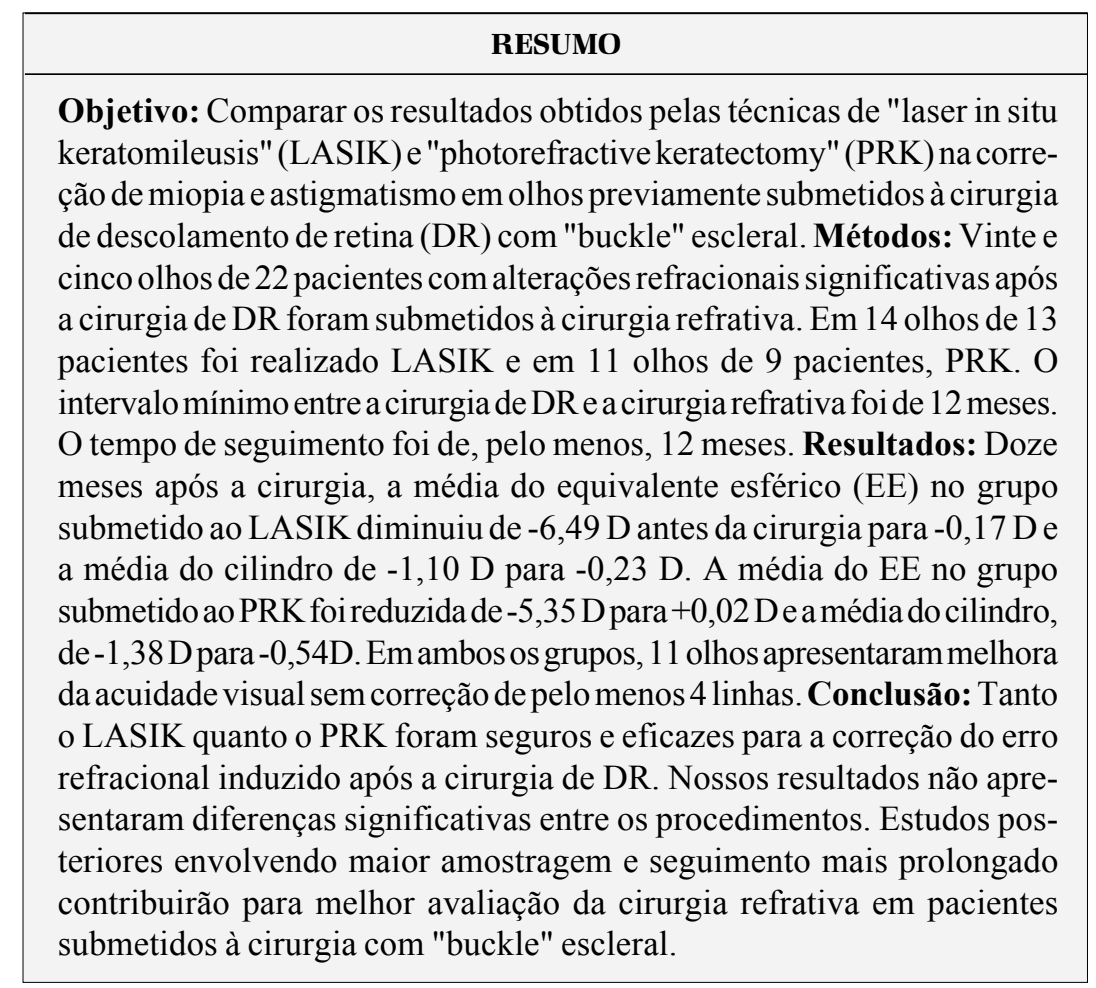

Descritores: Ceratectomia fotorrefrativa por excimer laser; Ceratomileuse assistida por excimer laser in situ; Deslocamento retiniano/cirurgia; Miopia/cirurgia; Astigmatismo/ cirurgia; Adulto

\section{INTRODUÇÃOO}

A indentação escleral por meio da colocação de faixa e explante escleral é um dos procedimentos utilizados rotineiramente para retinopexia em pacientes com descolamento de retina simples. Consiste na aproximação mecânica do epitélio pigmentado da retina sensorial selando a rasgadura que permite a entrada de líquido sub-retiniano ${ }^{(1)}$. Esse procedimento leva a uma compressão do equador do bulbo ocular com mudança em seu comprimento axial. De acordo com a literatura há aumento do diâmetro ântero-posterior do olho de cerca de $0,99 \mathrm{~mm}$ com indução de miopia em torno de $2,75 \mathrm{D}$, podendo também, ocorrer astigmatismo regular ou irregular ${ }^{(2-3)}$.

LASIK e PRK constituem atualmente os principais procedimentos para tratamento de ametropias ${ }^{(4-5)}$. As indicações para realização de cirurgia refrativa com o "excimer laser" não se limitam apenas à correção do erro refracional bilateral, sendo também utilizado para o tratamento de anisometropias em que o paciente não tolera a correção com óculos ou lentes de contato. Em pacientes submetidos à cirurgia com "buckle" escleral, o erro refracional induzido pode comprometer o resultado cirúrgico, principalmente quando 
houver anisometropia. Nestes casos a cirurgia refrativa pode ser indicada, favorecendo a recuperação da visão binocular.

Devido a algumas vantagens do LASIK sobre o PRK, tais como: recuperação mais rápida da acuidade visual, menor risco de "haze", maior conforto pós-operatório e melhor resultado em altos erros refracionais, o LASIK tem sido a técnica de primeira escolha para correção de erros refrativos. Porém, na técnica LASIK, o olho é mais manipulado que no PRK.

A realização de cirurgias refrativas a laser em olhos submetidos à correção de descolamento de retina pode induzir a novo descolamento devido à manipulação ocular, o que pode ser agravado pela onda de choque liberada pelo laser ${ }^{(6-8)} . \mathrm{Na}$ técnica LASIK o aumento súbito da pressão ocular provocado pelo anel de sucção durante a confecção do "flap" aumenta ainda mais o risco de novas rasgaduras na retina. $\mathrm{O}$ objetivo do presente trabalho é comparar a eficácia e segurança do LASIK e PRK em pacientes previamente operados com "buckle" escleral.

\section{MÉTODOS}

Foram avaliados 25 olhos de 22 pacientes submetidos à retinopexia com "buckle" escleral para o tratamento de descolamento de retina regmatogênico simples, e que apresentaram erro refracional significativo induzido pela cirurgia. Em 14 olhos de 13 pacientes ( 9 homens e 4 mulheres) foi realizado LASIK e em 11 olhos, o PRK ( 5 homens e 4 mulheres).

O tempo mínimo entre a retinopexia e a cirurgia refrativa foi de 12 meses e o seguimento pós-operatório após a cirurgia refrativa foi também de, pelo menos, 12 meses. Todos os pacientes foram informados sobre os procedimentos, técnicas, riscos, complicações e outras alternativas ao tratamento com a cirurgia refrativa, e assinaram um termo de consentimento antes da cirurgia.

A técnica de Custodis, modificada por Lincoff, foi utilizada para a cirurgia de descolamento de retina. Inicialmente foi obtida midríase através da aplicação tópica de tropicamida $1 \%$ $\left(\right.$ Mydriacyl $^{\circledR}$ ) e fenilefrina 10\% (Fenilefrina ${ }^{\circledR}$ ) com intervalo de 5 minutos, sendo iniciados 30 minutos antes da anestesia peribulbar. É feita peritomia $360^{\circ}$, reparo dos músculos retos horizontais e verticais, passagem de explante $277 \mathrm{em} 360^{\circ}$ sob os músculos retos que é fixado com fio Mercilene 5-0, e passagem da faixa 240, ambos de silicone duro, levando a introflexão das regiões das roturas. Essa técnica inclui ainda crioterapia e pneumoretinopexia com hexafluoreto de enxofre $\left(\mathrm{SF}_{6}\right)$ ou perfluorpropano $\left(\mathrm{C}_{3} \mathrm{~F}_{8}\right)$. A conjuntiva é fechada com Vycril 7-0 e dexametasona $10 \mathrm{mg}$ (Decadron ${ }^{\circledR}$ ) é infundida via subconjuntival, seguida de oclusão por 24 horas com pomada de acetato de retinol, aminoácidos, metionina e cloranfenicol (Epitezan $\left.{ }^{\circledR}\right)$. O paciente é orientado a permanecer em repouso absoluto com a cabeça posicionada de acordo com a localização da rasgadura retiniana. No pós-operatório todos os pacientes utilizaram colírios de tobramicina $0,3 \%$ e fosfato de dexametasona sódica $0,1 \%\left(\right.$ Tobradex $^{\circledR}$ ) de $3 / 3 \mathrm{~h}$ e tropicamida $1 \%$ de $6 / 6$ h por 15 dias, além de anti-inflamatório e analgésico oral (Diclofenaco de sódio $50 \mathrm{mg}$ ) de $8 / 8 \mathrm{~h}$ por 5 dias.

Com 1, 3, 7, 14, 28, 45 e 60 dias e 3, 6 e 12 meses os pacientes foram avaliados através de oftalmoscopia binocular indireta, tonometria de aplanação e biomicroscopia. Após o $45^{\circ}$ dia foi também medida a acuidade visual sem e com correção.

Os pacientes incluídos no estudo apresentavam anisometropia significativa induzida pela retinopexia, e acuidade visual com correção mínima de 20/200. Foram excluídos os pacientes portadores de doença auto-imune ou infecto-contagiosa e "diabetes mellitus".

Os exames pré-operatórios para a cirurgia refrativa incluíram: histórico médico ocular, medidas da acuidade visual sem e com correção, refração sob cicloplegia, biomicroscopia, paquimetria, tonometria, ceratometria, topografia corneana, microscopia especular da córnea e oftalmoscopia binocular indireta. Portadores de degenerações periféricas da retina foram submetidos a fotocoagulação com laser de argônio pelo menos quinze dias antes da cirurgia refrativa, quando indicada.

Os procedimentos cirúrgicos para LASIK e PRK foram realizados no Instituto de Olhos de Goiânia utilizando-se o excimer laser Technolas 217-C (Chiron Technolas/Bausch \& Lomb). Em todos os casos a programação foi visando a emetropia.

Todos os pacientes submetidos ao PRK, apresentavam alguma irregularidade importante na conjuntiva, secundária à cirurgia de descolamento de retina, que dificultava o posicionamento do anel de sucção.

O valor do erro refracional a ser corrigido, tamanho da zona óptica e paquimetria foram inseridos no programa do computador do aparelho.

Os pacientes submetidos à técnica LASIK receberam sedativo oral (Diazepan ${ }^{\circledR} 5 \mathrm{mg}$ ) no pré-operatório, e 2 gotas de colírio anestésico tópico (proparacaína 0,5\%) aplicadas entre 2 e 5 minutos antes da cirurgia. Campo plástico estéril e blefarostato foram colocados. A córnea foi marcada com caneta de violeta de genciana para garantir o perfeito reposicionamento do disco corneano ao término da cirurgia. $\mathrm{O}$ anel de sucção foi posicionado e o pedal pressionado elevando-se a pressão ocular até cerca de $65 \mathrm{mmHg}$, que foi confirmada com tonômetro de Barraquer. Utilizou-se o microcerátomo ACS (Chiron Technolas/Bausch \& Lomb) para a confecção do disco corneano de 8,5 mm de diâmetro e 160 um de espessura. O paciente foi orientado a fixar a luz coaxial do laser, que é focado e centrado na pupila. A pupila é iluminada com uma luz infravermelha e o sistema "eye tracker" acionado. O disco é rebatido nasalmente com uma espátula apropriada e a ablação corneana com laser é realizada no leito estromal. Em seguida, o leito estromal de acordo com a marcação prévia da caneta de violeta de genciana, é irrigado com solução salina balanceada e o disco reposicionado sem suturas; após 3 minutos de secagem é instilada 1 gota de Tobradex ${ }^{\circledR}$ colírio no fundo de saco. O olho é coberto com protetor de plástico transparente que é fixado com esparadrapo. Todos os pacientes utilizaram no pós-operatório Tobradex ${ }^{\circledR}$ de $6 / 6$ h por 1 semana. Em casos de 
alteração do filme lacrimal sintomática foi utilizada lágrima artificial sem preservativo.

Os pacientes submetidos à PRK receberam sedativo oral (Diazepan ${ }^{\circledR} 5 \mathrm{mg}$ ) no pré-operatório, e 2 gotas de colírio anestésico tópico (proparacaína 0,5\%) aplicadas entre 2 e 5 minutos antes da cirurgia. Campo plástico estéril e blefarostato foram colocados. Através do microscópico foi determinado o centro pupilar. O foco do laser foi ajustado precisamente na superfície do epitélio corneano e centrado na pupila. Em seguida o epitélio foi removido mecanicamente com espátula de ponta romba. Assim como na técnica de LASIK, o paciente foi orientado a fixar a luz coaxial do laser e o sistema "eye tracker" acionado e procedeu-se a ablação corneana. No pós-operatório utilizou-se lente de contato terapêutica por 4 dias, Tobra$\operatorname{dex}^{\circledR}$ e cetorolaco de trometamina $\left(\right.$ Acular $^{\circledR}$ ) de $4 / 4$ h por 1 semana. Também para esta técnica, em casos com alteração do filme lacrimal sintomática, foi utilizada lágrima artificial sem preservativo.

Todos os pacientes foram examinados após 1 e 7 dias com o biomicroscópio. Após 1, 3, 6, 9 e 12 meses os exames incluíram: medida da acuidade visual, topografia corneana, refração dinâmica e estática, tonometria e oftalmoscopia binocular indireta. Foi questionado aos pacientes sintomas como "glare", halos, flutuação da visão, fotofobia e irritação.

Todas as cirurgias de descolamento de retina foram feitas pelo mesmo cirurgião especialista em retina e vítreo (J.J.N.J.) e as refrativas pela mesma cirurgiã especialista em córnea e cirurgia refrativa (B.A.N.).

\section{RESULTADOS}

Nos pacientes operados pela técnica LASIK a média do equivalente esférico passou de $-6,49 \mathrm{D}(\mathrm{de}-10,87 \mathrm{a}-1,75 \mathrm{D})$ para $-0,18 \mathrm{D}(-1,25 \mathrm{a}+0,25 \mathrm{D})$ com 6 meses e para $-0,17 \mathrm{D}(-1,00 \mathrm{D}$ a plano) 12 meses após a cirurgia. Em relação aos pacientes submetidos ao PRK a média do equivalente esférico no pré-operatório foi de $-5,35 \mathrm{D}(-8,50 \mathrm{a}-2,75 \mathrm{D})$ diminuindo para $+0,12 \mathrm{D}(-0,75 \mathrm{a}$
$+0,75 \mathrm{D})$ com 6 meses, e após 12 meses para +0,02 D (-1,00 a $+0,87$ D) (Tabela 1).

A média do cilindro pré-operatória reduziu de $-1,10 \mathrm{D}$ (zero a -3,00 D) para -0,28 D (zero a -0,75 D) com 6 meses e para -0,23 D (zero a -0,75 D) aos 12 meses nos pacientes submetidos a LASIK. Nos pacientes submetidos ao PRK a média do cilindro diminui de -1,38 D (zero a -3,00 D) para-0,43 D (zero a -0,75 D) com 6 meses e para -0,54 D (zero a -1,00 D) após 12 meses (Tabela 2).

Em ambos os procedimentos houve melhora da acuidade visual sem correção em todos os olhos, medida com optotipos de Snellen. Nos pacientes submetidos a LASIK houve melhora de pelo menos 4 linhas em 11 olhos (78,57\%) sem correção; em relação à acuidade visual com correção, permaneceu estável em 6 olhos $(42,85 \%)$ e melhorou nos demais. Quanto ao PRK, o ganho mínimo de 4 linhas sem correção ocorreu em todos os olhos, e a acuidade com correção manteve-se estável em 6 olhos $(54,54 \%)$, com melhora em 5 olhos $(45,45 \%)$ (Tabela 3 e 4).

Doze meses após o LASIK, 12 olhos (86\%) apresentavam córneas claras e transparentes e o limite do "flap" era visto com dificuldade à biomicroscopia. Em 2 casos (casos 8 e 11) observou-se piora de 1 linha quando comparada a acuidade com correção prévia e a sem correção do pós-operatório, sendo nesses evidenciado discreto "haze" corneano. Porém quando comparados com a acuidade visual pós-operatória corrigida não apresentava perda de linha pela tabela de Snellen.

Queixa de dor e sensação de corpo estranho ocorreu em todos os pacientes submetidos ao PRK nos 4 primeiros dias pós-operatórios, apesar do uso da lente de contato terapêutica e do Acular ${ }^{\circledR}$. Após o PRK, 3 pacientes (casos 2, 6 e 10) apresentaram "haze" corneano (1 a $2+$ em 4 ). Dois pacientes (casos 6 e 9) tiveram piora de 1 linha quando comparada a acuidade com correção antes e a sem correção após a cirurgia. Porém quando comparados com a acuidade visual pós-operatória corrigida não apresentava perda de linha pela tabela de Snellen.

Em nenhum caso foi necessário reoperação para correção

\begin{tabular}{|c|c|c|c|c|c|c|c|c|}
\hline \multirow[t]{2}{*}{ Caso № } & \multicolumn{4}{|c|}{ LASIK } & \multicolumn{4}{|c|}{ PRK } \\
\hline & Pré-op. & 3 meses & 6 meses & 12 meses & Pré-op. & 3 meses & 6 meses & 12 meses \\
\hline 1 & $-10,87$ & $+0,75$ & Plano & Plano & $-6,37$ & $-0,25$ & Plano & $-0,25$ \\
\hline 2 & $-10,12$ & $+0,75$ & $+0,25$ & Plano & $-5,75$ & $-0,87$ & $-0,75$ & $-1,00$ \\
\hline 3 & $-6,75$ & $-1,00$ & $-1,00$ & $-0,75$ & $-4,75$ & $+0,63$ & $+0,38$ & $+0,25$ \\
\hline 4 & $-10,12$ & $-0,50$ & Plano & $-0,25$ & $-4,50$ & $-0,37$ & $-0,25$ & $-0,37$ \\
\hline 5 & $-7,50$ & Plano & $-0,25$ & $-0,62$ & $-6,62$ & $+0,63$ & $+0,25$ & +025 \\
\hline 6 & $-6,25$ & Plano & Plano & Plano & $-7,00$ & $-1,00$ & $-0,75$ & $-0,75$ \\
\hline 7 & $-7,50$ & $+0,50$ & $+0,25$ & $-0,25$ & $-2,75$ & $+1,00$ & $+0,75$ & $+0,50$ \\
\hline 8 & $-3,25$ & $-0,50$ & $-0,37$ & $-0,37$ & $-3,62$ & $+0,75$ & $+0,50$ & $+0,50$ \\
\hline 9 & $-4,75$ & $-0,25$ & Plano & Plano & $-3,25$ & Plano & $-0,12$ & $-0,50$ \\
\hline 10 & $-5,00$ & Plano & Plano & Plano & $-8,50$ & $+1,00$ & $+0,75$ & $+0,75$ \\
\hline 11 & $-6,50$ & $-0,50$ & $-0,25$ & $-0,25$ & $-5,75$ & $+0,75$ & $+0,63$ & $+0,87$ \\
\hline 12 & $-6,37$ & $-0,12$ & Plano & Plano & - & - & - & - \\
\hline 13 & $-1,75$ & Plano & Plano & Plano & - & - & - & - \\
\hline 14 & $-4,25$ & $-1,25$ & $-1,25$ & $-1,00$ & - & - & - & - \\
\hline
\end{tabular}




\begin{tabular}{|c|c|c|c|c|c|c|c|c|}
\hline \multirow[t]{2}{*}{ Caso № } & & & & & & & & \\
\hline & Pré-op. & 3 meses & 6 meses & 12 meses & Pré-op. & 3 meses & 6 meses & 12 meses \\
\hline 1 & $-0,25 \times 110^{\circ}$ & Plano & Plano & Plano & $-0,75 \times 90^{\circ}$ & $-0,50 \times 85^{\circ}$ & $-0,50 \times 90^{\circ}$ & $-0,50 \times 90^{\circ}$ \\
\hline 2 & $-0,75 \times 120^{\circ}$ & Plano & Plano & Plano & $-1,50 \times 115^{\circ}$ & $-0,75 \times 110^{\circ}$ & $-0,50 \times 100^{\circ}$ & $-1,00 \times 115^{\circ}$ \\
\hline 3 & $-1,00 \times 90^{\circ}$ & $-0,25 \times 90^{\circ}$ & $-0,50 \times 90^{\circ}$ & $-0,50 \times 90^{\circ}$ & $-2,00 \times 45^{\circ}$ & $-0,75 \times 70^{\circ}$ & $-0,75 \times 70^{\circ}$ & $-1,00 \times 60^{\circ}$ \\
\hline 4 & $-0,25 \times 10^{\circ}$ & Plano & Plano & Plano & $-0,50 \times 30^{\circ}$ & $-0,75 \times 180^{\circ}$ & $-0,50 \times 180^{\circ}$ & $-0,75 \times 180^{\circ}$ \\
\hline 5 & $-1,00 \times 25^{\circ}$ & $-1,00 \times 25^{\circ}$ & $-0,75 \times 25^{\circ}$ & $-0,75 \times 25^{\circ}$ & $-2,25 \times 10^{\circ}$ & $-0,75 \times 170^{\circ}$ & $-0,50 \times 170^{\circ}$ & $-0,50 \times 170^{\circ}$ \\
\hline 6 & Plano & Plano & Plano & Plano & Plano & Plano & Plano & Plano \\
\hline 7 & $-1,50 \times 180^{\circ}$ & $-0,50 \times 180^{\circ}$ & $-0,50 \times 180^{\circ}$ & $-0,50 \times 180^{\circ}$ & $-1,00 \times 75^{\circ}$ & Plano & Plano & Plano \\
\hline 8 & $-2,00 \times 130^{\circ}$ & $-0,75 \times 40^{\circ}$ & $-0,75 \times 30^{\circ}$ & $-0,75 \times 30^{\circ}$ & $-1,75 \times 05^{\circ}$ & $-0,50 \times 180^{\circ}$ & $-0,50 \times 180^{\circ}$ & $-0,50 \times 10^{\circ}$ \\
\hline 9 & Plano & Plano & Plano & Plano & $-3,00 \times 130^{\circ}$ & $-0,75 \times 145^{\circ}$ & $-0,75 \times 145^{\circ}$ & $-1,00 \times 145^{\circ}$ \\
\hline 10 & $-2,00 \times 80^{\circ}$ & $-0,25 \times 80^{\circ}$ & Plano & Plano & $-1,00 \times 10^{\circ}$ & Plano & Plano & Plano \\
\hline 11 & $-3,00 \times 180^{\circ}$ & $-0,50 \times 160^{\circ}$ & $-0,50 \times 160^{\circ}$ & $-0,50 \times 160^{\circ}$ & $-1,50 \times 180^{\circ}$ & $-0,50 \times 170^{\circ}$ & $-0,75 \times 170^{\circ}$ & $-0,75 \times 170^{\circ}$ \\
\hline 12 & $-1,75 \times 10^{\circ}$ & $-0,25 \times 110^{\circ}$ & Plano & Plano & - & - & - & - \\
\hline 13 & Plano & Plano & Plano & Plano & - & - & - & - \\
\hline 14 & $-2,00 \times 145^{\circ}$ & $-0,50 \times 145^{\circ}$ & $-0,50 \times 145^{0}$ & $-0,50 \times 145^{\circ}$ & - & - & - & - \\
\hline
\end{tabular}

do erro refracional residual. Não foram encontradas alterações retinianas durante todo o seguimento de ambas as técnicas.

A topografia corneana não evidenciou descentração da ablação em nenhum caso. Em todos os olhos tivemos uma diminuição do poder dióptrico da córnea, compatível com a mudança na refração.

Antes das cirurgias refrativas, a média da contagem endotelial foi $2152 \pm 150$ células $/ \mathrm{mm}^{2}\left(1200\right.$ a 2500 células $\left./ \mathrm{mm}^{2}\right)$ no grupo submetido ao LASIK e $2264 \pm 452$ células $/ \mathrm{mm}^{2}(1670$ a 2890 células $/ \mathrm{mm}^{2}$ ) no grupo do PRK. Aos 6 meses, $2140 \pm 110$ células $/ \mathrm{mm}^{2}$ (1270 a 2540 células $/ \mathrm{mm}^{2}$ ) para o LASIK e $2265 \pm$ 440 células $/ \mathrm{mm}^{2}$ (1701 a 2910 células $/ \mathrm{mm}^{2}$ ) no PRK; após 12 meses, a média foi de $2120 \pm 156$ células $/ \mathrm{mm}^{2}(1340$ a 2550 células $/ \mathrm{mm}^{2}$ ) para o LASIK e $2246 \pm 445$ células $/ \mathrm{mm}^{2}$ (1638 a 2860 células $/ \mathrm{mm}^{2}$ ) no PRK.

Em relação à pressão ocular, a média foi de 12,00 $\pm 1,50$ $\mathrm{mmHg}(11 \mathrm{a} 14 \mathrm{mmHg}$ ) antes do LASIK e de $11,18 \pm 1,34 \mathrm{mmHg}$ (10 a $14 \mathrm{mmHg}$ ) antes do PRK; aos 6 meses, $11,10 \pm 1,00 \mathrm{mmHg}$ (10 a $13 \mathrm{mmHg}$ ) no LASIK e 10,36 $\pm 0,92 \mathrm{mmHg}$ (9 a $12 \mathrm{mmHg}$ ) no PRK; aos 12 meses a média foi de $12,70 \pm 0,90 \mathrm{mmHg}(11$ a 13 $\mathrm{mmHg}$ ) no LASIK e de 10,27 $\pm 1,18 \mathrm{mmHg}$ (9 a $13 \mathrm{mmHg}$ ).
A espessura corneana antes do LASIK apresentava valor médio de $568 \pm 34 \mu \mathrm{m}(525$ a $598 \mu \mathrm{m})$, e $537 \pm 26 \mu \mathrm{m}$ (502 a 583 $\mu \mathrm{m})$ antes do PRK. Após 6 meses essa média foi de $438 \pm 36 \mu \mathrm{m}$ $(390 \mathrm{a} 484 \mu \mathrm{m})$ para o LASIK e $425 \pm 32 \mu \mathrm{m}(386 \mathrm{a} 480 \mu \mathrm{m})$ para o PRK; com 12 meses, $440 \pm 33 \mu \mathrm{m}(392$ a $492 \mu \mathrm{m})$ para o LASIK e $425 \pm 31 \mu \mathrm{m}$ (390 a $484 \mu \mathrm{m})$ para o PRK. A ablação corneana variou de 75 a $160 \mu \mathrm{m}$ no grupo do LASIK e de 45 a $140 \mu \mathrm{m}$ no grupo do PRK.

\section{DISCUSSÃO}

O LASIK e PRK são cirurgias utilizadas freqüentemente com eficácia para correção de erros refrativos ${ }^{(5,9)}$. Alguns autores questionam a segurança desses procedimentos para o seguimento posterior, referindo até a ocorrência de descolamento de retina e rasgaduras ${ }^{(6-8)}$. Os mecanismos comuns a ambas as técnicas que explicariam essas complicações seriam a manipulação ocular e a onda de choque provocada pelo laser em ambas as técnicas. No LASIK o fator agravante seria principalmente o aumento e descompressão súbitos da pressão

\begin{tabular}{|c|c|c|c|c|c|c|c|c|c|}
\hline \multicolumn{10}{|c|}{ Tabela 3. Dados dos pacientes submetidos a LASIK } \\
\hline \multirow[t]{2}{*}{ Caso № } & \multicolumn{5}{|c|}{ Pré-operatório } & \multicolumn{4}{|c|}{ Pós-operatório (12 meses) } \\
\hline & $\overline{T R}$ & Avsc & AVcc & & Refração & Refração & AVsc & AVcc & Ganho AV \\
\hline 1 & 12 & $20 / 800$ & $20 / 100$ & $-10,75$ & $-0,25 \times 110^{\circ}$ & Plano & $20 / 80$ & $20 / 70$ & 7 \\
\hline 2 & 14 & $20 / 600$ & $20 / 40$ & $-9,75$ & $-0,25 \times 120^{\circ}$ & Plano & $20 / 30$ & $20 / 20$ & 11 \\
\hline 3 & 12 & $20 / 400$ & $20 / 100$ & $-6,25$ & $-1,00 \times 900^{\circ}$ & $-0,50-0,50 \times 90^{\circ}$ & $20 / 100$ & $20 / 100$ & 4 \\
\hline 4 & 13 & $20 / 600$ & $20 / 150$ & $-10,00$ & $-0,25 \times 110^{\circ}$ & $-0,25$ & $20 / 150$ & $20 / 150$ & 4 \\
\hline $5^{\star}$ & 12 & $20 / 400$ & $20 / 200$ & $-7,00$ & $-1,00 \times 25^{\circ}$ & $-0,25-0,75 \times 25^{\circ}$ & $20 / 200$ & $20 / 200$ & 2 \\
\hline $6^{*}$ & 16 & $20 / 400$ & $20 / 100$ & $-6,25$ & & Plano & $20 / 80$ & $20 / 80$ & 5 \\
\hline 7 & 14 & $20 / 800$ & $20 / 200$ & $-6,75$ & $-1,50 \times 180^{\circ}$ & Plano $-0,50 \times 180^{\circ}$ & $20 / 200$ & $20 / 200$ & 4 \\
\hline 8 & 24 & $20 / 200$ & $20 / 50$ & $-2,25$ & $-2,00 \times 130^{\circ}$ & Plano $-0,75 \times 30^{\circ}$ & $20 / 60$ & $20 / 40$ & 5 \\
\hline 9 & 12 & $20 / 400$ & $20 / 100$ & $-4,75$ & & Plano & $20 / 80$ & $20 / 80$ & 5 \\
\hline 10 & 12 & $20 / 800$ & $20 / 200$ & $-4,00$ & $-2,00 \times 80^{\circ}$ & Plano & $20 / 200$ & $20 / 200$ & 4 \\
\hline 11 & 12 & $20 / 400$ & $20 / 150$ & $-5,00$ & $-3,00 \times 150^{\circ}$ & Plano $-0,50 \times 160^{\circ}$ & $20 / 200$ & $20 / 100$ & 2 \\
\hline 12 & 13 & $20 / 400$ & $20 / 100$ & $-5,50$ & $-1,75 \times 10^{\circ}$ & Plano & $20 / 100$ & $20 / 100$ & 4 \\
\hline 13 & 14 & $20 / 200$ & $20 / 80$ & $-1,75$ & & Plano & $20 / 70$ & $20 / 70$ & 4 \\
\hline 14 & 16 & $20 / 400$ & $20 / 200$ & $-3,25$ & $-2,00 \times 145^{\circ}$ & $-0,75-0,50 \times 145^{\circ}$ & $20 / 150$ & $20 / 150$ & 3 \\
\hline
\end{tabular}




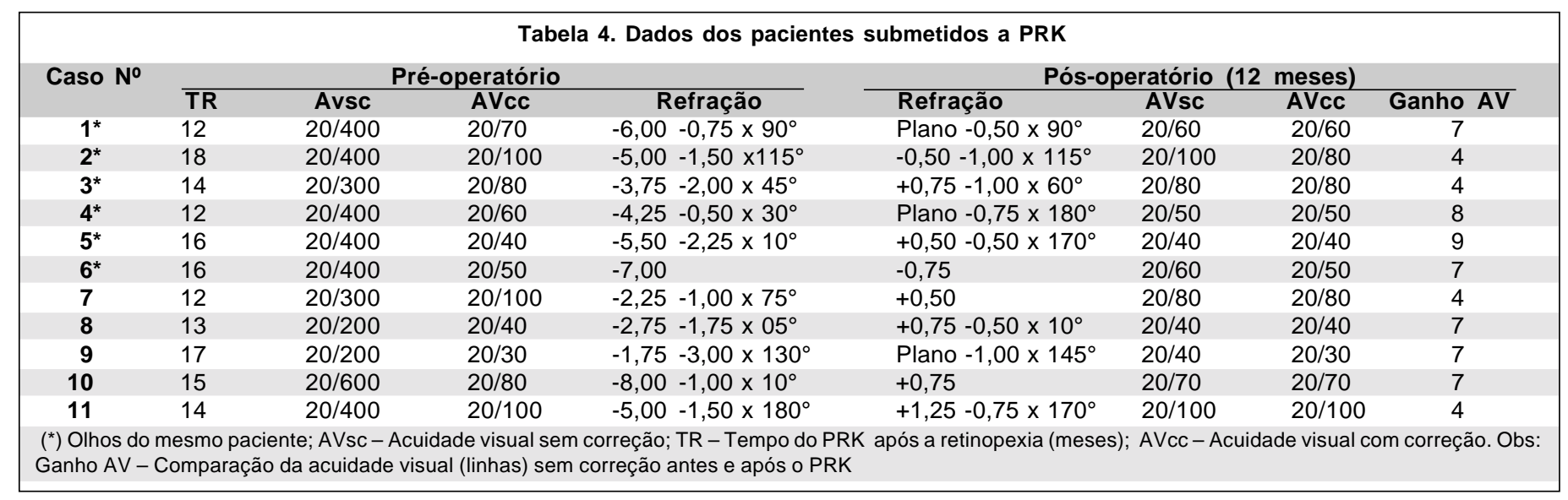

ocular provocada pelo anel de sucção, podendo levar à tração vítrea $^{(4,6,10-11)}$.

Alguns pacientes com cirurgia ocular prévia, como no caso da cirurgia de descolamento de retina com "buckle" escleral, podem apresentar irregularidades conjuntivais causadas pela cicatrização que dificultam a adaptação do anel de sucção. Isto pode inviabilizar a técnica LASIK ou aumentar o risco de complicações durante a cirurgia, tais como a perda da sucção para a confecção do "flap". Nestes casos, o PRK pode ser a melhor opção para a correção refrativa $a^{(9,11)}$.

Em estudo anterior comprovamos a eficácia do LASIK no tratamento de pacientes portadores de miopia e astigmatismo previamente submetidos à cirurgia de descolamento de retina com "buckle" escleral ${ }^{(11)}$. Neste trabalho, comparamos os resultados entre as técnicas LASIK e PRK com o objetivo de avaliar sua eficácia, previsibilidade, estabilidade e segurança. Após os procedimentos não foram evidenciadas alterações fundoscópicas. Ambas as técnicas foram eficazes na correção da ametropia, tendo sido alcançada a eliminação ou redução do erro refracional, favorecendo a visão binocular.

Dois casos apresentaram piora da acuidade visual sem correção quando comparadas com a acuidade visual com correção no pré-operatório, com perda de 1 linha da tabela de Snellen, em ambas as técnicas. Isso pode ser explicado pela persistência de erro refrativo residual, que quando corrigido, permite que o paciente apresente a mesma acuidade (PRK) ou ganho de 1 linha (LASIK) quando comparamos com a acuidade pré-operatória, apesar da ocorrência de "haze" em 3 pacientes.

\section{CONCLUSÃO}

Concluímos que em todos os casos em que utilizamos o PRK, quando comparados ao LASIK, todos foram eficazes para correção do erro refrativo e não apresentaram qualquer complicação para o seguimento posterior.

Ao considerarmos que os pacientes avaliados em nosso estudo apresentaram patologia retiniana prévia importante, e a ausência de alterações no exame fundoscópico pós-operatório, destacamos a importância de uma cuidadosa manipulação do bulbo ocular durante o procedimento cirúrgico e o domínio da técnica proposta. No caso do LASIK, o tempo de sucção, quando a pressão intra-ocular está aumentada, deve ser reduzido ao máximo, diminuindo assim um dos fatores que poderiam desencadear complicações no pólo posterior, objetivando o permanente sucesso da cirurgia e a satisfação do paciente. Nos casos em que o LASIK não puder ser utilizado, o PRK constitui uma opção segura e eficaz para o tratamento dessas ametropias.

\section{ABSTRACT}

Purpose: To compare the results of "laser in situ keratomileusis" (LASIK) and "photorefractive keratectomy" (PRK) to correct myopia and astigmatism in eyes previously submitted to retinal detachment (RD) surgery with scleral "buckle". Methods: Twenty-five eyes of 22 patients submitted to scleral buckling procedure with significant refraction changes after surgery were evaluated. Fourteen eyes of 13 patients underwent LASIK and 11 eyes of 9 patients underwent PRK. Minimum time between RD surgery and refractive surgery was 12 months. All patients were followed-up for at least 12 months. Results: Twelve months after surgery, mean spherical equivalent in the LASIK group changed from $-6.49 \mathrm{D}$ preoperatively to $-0.17 \mathrm{D}$ and mean astigmatism decreased from $-1.10 \mathrm{D}$ to $-0.23 \mathrm{D}$. Spherical equivalent in the PRK group decreased from $-5.35 \mathrm{D}$ before surgery to $+0,02 \mathrm{D}$ and average astigmatism changed from $-1.38 \mathrm{D}$ to $-0.54 \mathrm{D}$. In both groups, uncorrected visual acuity (UCVA) improved at least 4 lines in 11 eyes. Conclusion: Both LASIK and PRK were safe and effective to correct refractive error after RD surgery. Our results showed no significant difference between procedures. Further studies with more patients and long-term follow-up are required to better evaluate the effects of refractive surgery in patients submitted to scleral buckling procedure.

Keywords: Keratomileusis laser in situ; Keratectomy photorefractive excimer laser; Retinal detachment/surgery; Myopia/ surgery; Astigmatism/surgery; Adult 


\section{REFERÊNCIAS}

1. Ryan SJ. Retina. $2^{\text {nd }}$ ed. St. Louis: Mosby; 1994. p.1979-2017

2. Smiddy WE, Loupe DN, Michels RG, Enger C, Glaser BM, deBustros S. Refractive changes after scleral buckling surgery. Arch Ophthalmol 1989; 107:1469-71.

3. Lancaster W. Refraction and motility. Springfield: Charles C. Thomas; 1952. p.149.

4. Nassaralla BR, Nassaralla JJ. Laser in situ keratomileusis after penetrating keratoplasty. J Refract Surg 2000;16:431-7.

5. Hersh PS, Brint SF, Maloney RK, Durrie DS, Gordon M, Michelson MA, Thompson VM, Berkeley RB, Schein OD, Steinert RF. Photorefractive kera- tectomy versus laser in situ keratomileusis for moderate to high myopia. A randomized prospective study. Ophthalmology 1998;105:1512-23.

6. Kim WS, Kim JS. Change in corneal sensitivity following laser in situ keratomileusis. J Cataract Refract Surg 1999;25:368-73.

7. Ruiz-Moreno JM, Pérez-Santonja JJ, Alió JL. Retinal detachment in myopic eyes after laser in situ keratomileusis. Am J Ophthalmol 1999;128:588-94.

8. Ruiz-Moreno JM, Artola A, Alió JL. Retinal detachment in myopic eyes after photorefractive keratectomy. J Cataract Refract Surg 2000;26:340-4.

9. Azar DT, Farah SG. Laser in situ keratomileusis versus photorefractive keratectomy: an update on indications and safety. Ophthalmology 1998;105:1357-8.

10. Barraquer JI. Cirurgia Refrativa de la Cornea. Bogota. O. P. Graficas, 1989.

11. Nassaralla Junior JJ, Nassaralla BA. LASIK após cirurgia para descolamento de retina. Rev Bras Oftalmol 2001;60:243-9.

\section{Simpósio Anual da Associação Paranaense de Offalmologia}

\section{Fórum Sul-Brasileiro de Complicações em Adapłação de Lenłes de Conłało}

\section{2 a 14 de Junho de 2003 \\ Hotel Bourbon - Curitiba - PR}

INFORMAC̣ÕES: Tel./Fax: (41) 232-4031

e-mail: apoftalmo@onda.com.br 